\title{
Water Wetting Observation on a Superhydrophobic Hairy Plant Leaf Using Environmental Scanning Electron Microscopy
}

\author{
Sun Mi Yoon ${ }^{1,2}$, Tae-Jun Ko ${ }^{1,3}$, Kyu Hwan $\mathrm{Oh}^{3}$, Sahn Nahm², Myoung-Woon Moon ${ }^{1, *}$ \\ ${ }^{1}$ Materials and Life Science Research Division, Korea Institute of Science and Technology, Seoul 02792, Korea \\ ${ }^{2}$ Department of Materials Science and Engineering, Korea University, Seoul 02841, Korea \\ ${ }^{3}$ Department of Materials Science and Engineering, Seoul National University, Seoul 08826, Korea
}

*Correspondence to:

Moon MW,

Tel: +82-2-958-5487

Fax: +82-2-958-5451

E-mail:mwmoon@kist.re.kr

Received December 7, 2016

Revised December 22, 2016

Accepted December 22, 2016
Functional surfaces in nature have been continuously observed because of their ability to adapt to the environment. To this end, methods such as scanning electron microscopy (SEM) have been widely used, and their wetting functions have been characterized via environmental SEM. We investigated the superhydrophobic hairy leaves of Pelargonium tomentosum, i.e., peppermint-scented geranium. Their surface features and wettability were studied at multiple-scales, i.e., macro-, micro-, and sub-micro scales. The surfaces of the investigated leaves showed superhydrophobicity at the macro-, and micro-scales. The wetting or condensing behavior was studied for molecule-size water vapors, which easily adhered to the hairy surface owing to their significantly lower size in comparison to that of the surface.

Key Words: Environmental scanning electron microscopy, Wetting, Superhydrophobic plant, Condensation

\section{INTRODUCTION}

Mankind has been continuously learning from nature via careful observation in attempt to improve their own lives and to further overcome the environmental issues. In the last 10 years, researchers have focused their attention on the surface functions of parts of living organisms, such as gecko's feet, water strider's legs, and lotus leaves for various applications (e.g., self-cleaning surfaces, nano-micro robotics, and water harvesting) (Bhushan \& Her, 2010; Cheng et al., 2005; Cho \& Choi, 2008; Gao \& Jiang, 2004; Hansen \& Autumn, 2005; Neinhuis \& Barthlott, 1997). In particular, functional plant surfaces such as lotus or acacia leaves have been studied owing to their excellent characteristics of superhydrophobicity and self-cleaning, which are a result of their structures and surface materials (Cha et al., 2010; Cheng et al., 2005; Neinhuis \& Barthlott, 1997). For characterizing superhydrophobicity, one can use simple water droplet measurements on target surfaces or measure the squeezing pressure at the micro-scale.
Measurement of water condensation with water vapors is also a key indicator of robust superhydrophobicity at the nanoscale (Ko et al., 2012, 2015; Quéré, 2008; Shin et al., 2012; Varanasi et al., 2009).

When a water droplet is placed on a superhydrophobic plant leaf possessing nano- or micro-scale roughness like hair or bump-shapes (Fig. 1A), the droplet forms a nearly perfect spherical shape, rolls off, and cleans the leaf surface as shown in Fig. 1B. This self-cleaning and water-repellent behavior is typically attributed to the roughness of the surface and lowsurface-energy coatings such as the hydrophobic epicuticular wax crystalloid coating of lotus leaves. Superhydrophobicity has been commonly characterized by higher water contact angles (i.e., higher than $160^{\circ}$ ) and wetting angle hysteresis of drop rolling. However, because this method uses relatively large water droplets with millimeter-scale sizes, additional information on the quality of superhydrophobicity has been recently obtained. A robust superhydrophobic surface should be able to sustain high contact angles against condensation

(a) This is an open-access article distributed under the terms of the Creative Commons Attribution Non-Commercial License (http://creativecommons.org/licenses/by-nc/4.0) which permits unrestricted noncommercial use, distribution, and reproduction in any medium, provided the original work is properly cited.

Copyrights @ 2016 by Korean Society of Microscopy 
of small-sized droplets such as vaporized water. Lotus leaves exhibit low resistance to water condensation owing to the low aspect ratio (defined as the ratio of the height to the width of the structures, ca. 2 or 3) of the nanopillar structure geometries for nanopillar structures (Cha et al., 2010; Cheng et al., 2005; Rahmawan et al., 2010). The water condensation behavior of particular functional organic surfaces has been measured using environmental scanning electron microscopy (ESEM). ESEM has attracted considerable attention as a promising method for studying the interactions between materials and humidity (i.e., humid air and water at the nanoor molecular-scale) in various disciplines such as biology, food and life science, and materials science (Cheng et al., 2005; Donald, 2003; Esmaily et al., 2015; Jansson et al., 2016; Ko et al., 2015; Varanasi et al., 2009).

In this study, we propose a method to characterize superhydrophobic plant leaves with an extremely high aspect ratio using a water droplet release method and ESEM condensation experiments. We have used Pelargonium tomentosum (also known as peppermint-scented geranium) having a superhydrophobic surface, which contained nano-sized hair-like structures with an aspect ratio of more than 25 (Fig. 1AD). The surfaces of plants are known to have the trichome structures, which protect the plant from water and insects (Brewer \& Smith, 1997; Gorb \& Gorb, 2002).

\section{MATERIALS AND METHODS}

P. tomentosum leaves were obtained from a regional botanic garden. The morphologies of the leaf surfaces at different scales were observed using a digital camera (60D; Canon, Japan) and with a scanning electron microscope (Nova NanoSEM 200; FEI, USA). Prior to scanning electron microscopy (SEM) observations, the sample surfaces were coated with platinum thin films of ca. $10 \mathrm{~nm}$ for protecting the plant surface from electron charging; the surfaces were then observed via SEM at a power of $10 \mathrm{kV}$.

The wettability was observed for millimeter-scale water droplets using the sessile drop technique; $20 \mu \mathrm{L}$ of deionized water was gently deposited on the P. tomentosum leaf using a micro-syringe. The wettability of the micro-meter scale water droplets, which replicate mist or fog-scale droplet was evaluated in a custom-made humidity-controlled chamber. Water mist was supplied to the P. tomentosum leaf for 2 hours, sequential images were taken by a digital camera (60D).

The sub-micro scale water droplet condensation behavior was

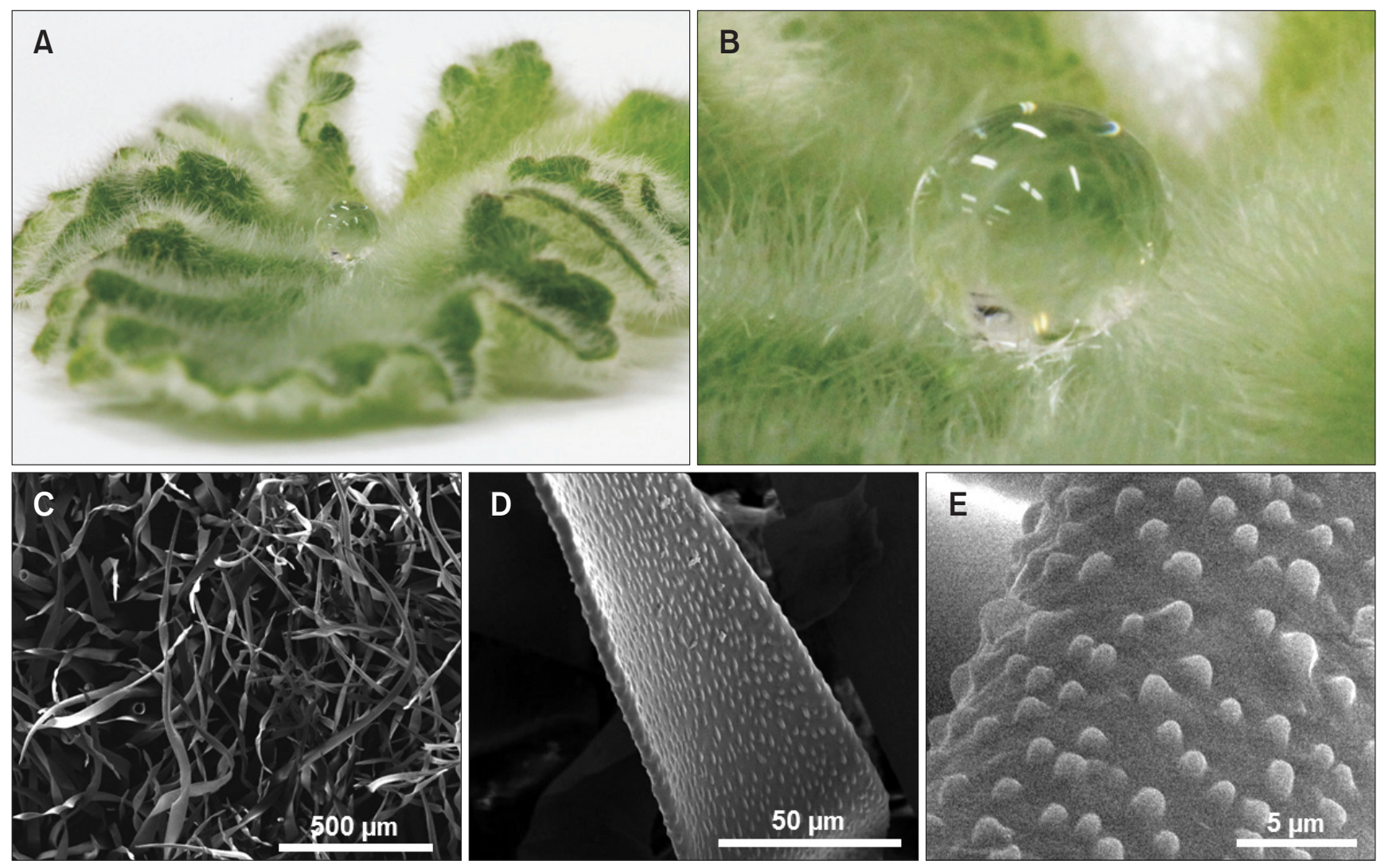

Fig. 1. (A) Pelargonium tomentosum leaf and (B) sessile water droplet on the P. tomentosum leaf. (C-E) Scanning electron microscopy images of the leaf surface. (C) Low-magnification picture of the surface, (D, E) morphology of trichome or hair on the P. tomentosum leaf. 
A
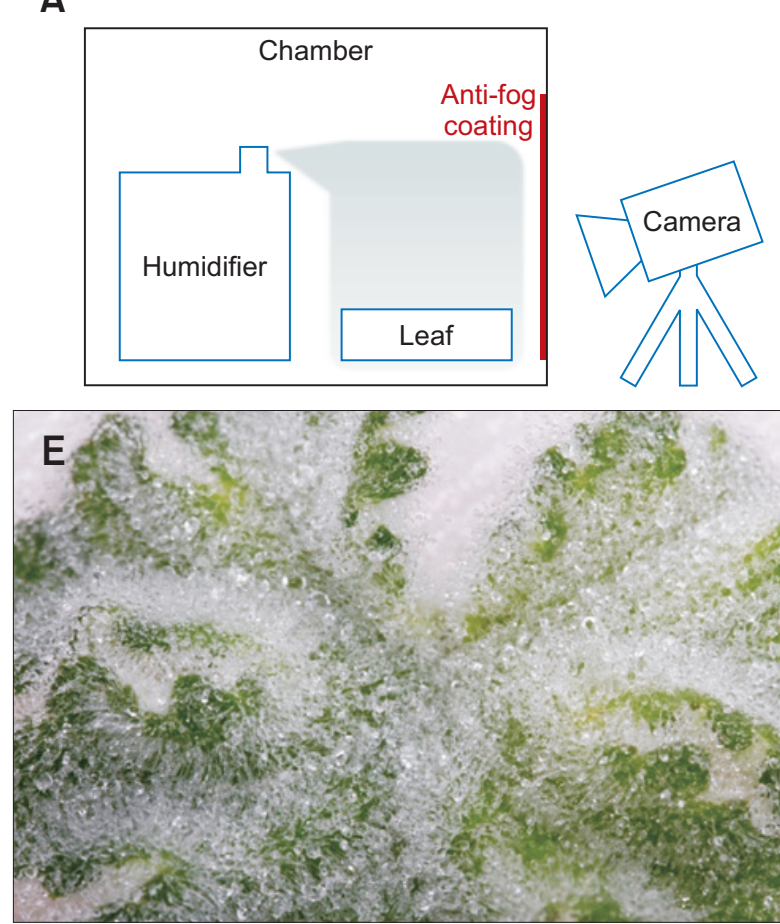

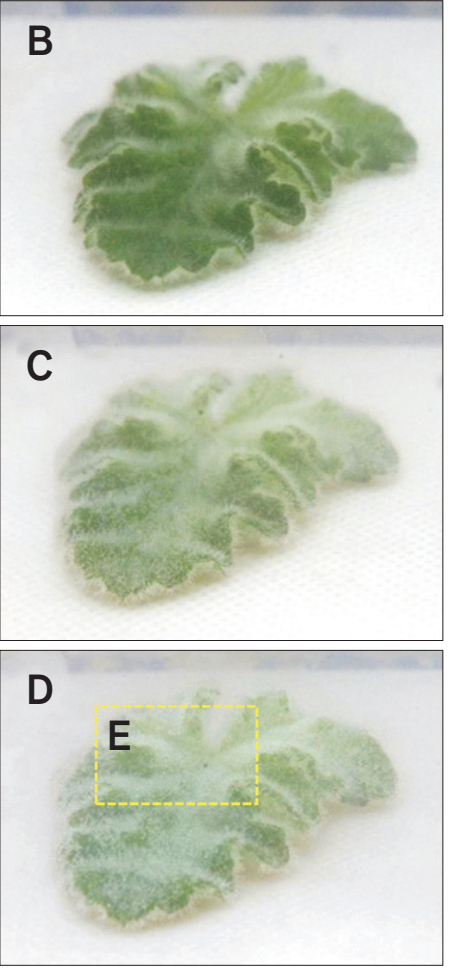

Fig. 2. (A) Custom-made humiditycontrolled chamber for the mist experiments. (B-E) Sequential photos of mist deposition on Pelargonium tomentosum leaves at: (B) 20, (C) 60, and (D, E) 120 minutes after the humidifier was connected. observed via ESEM (XL-30 FEG; FEI) at a power of $15 \mathrm{kV}$. A piece of the $P$. tomentosum leaf was placed on a Peltier cooling stage module, and the edge of the leaf was subsequently covered with silver paste to increase its thermal conductivity. The temperature was controlled at $2^{\circ} \mathrm{C}$ and chamber pressure was increased from 3.0 to 5.6 Torr. Images were taken with respect to the pressure-holding duration for 5 minutes during observation.

\section{RESULTS AND DISCUSSION}

Both sides of the whole leaf surface were covered with hair or trichome, as shown in Fig. 1A. The surface of the hairy leaf was observed using an electron microscope for detailed morphology observation. The hair or trichome contained ca. $50 \mu \mathrm{m}$ of the main body and $1 \mu \mathrm{m}$ of the bumped structure, as shown in Fig. 1D and E. According to a previous study, the leaf surface requires a hierarchical structure (i.e., nanostructures on microstructures) and hydrophobic wax components to achieve superhydrophobicity (Koch \& Barthlott, 2009). Millimeter-scale water droplets were hardly deposited as a result of the low adhesion between the water droplet and the hairy structure. Thus, the water droplets maintained their spherical shape, and the contact angle was $160^{\circ}$ (i.e., superhydrophobicity was achieved).

The wettability during condensation was determined using a micro-meter scale mist (Fig. 2). Droplets of micro-meter scale mist were deposited on a P. tomentosum leaf; with time, the size of these droplets started to grow. After 2 hours of mist deposition, the deposited mist droplets maintained a spherical shape (Fig. 2E), which indicated that the P. tomentosum leaf was hardly wet by micro-meter scale mist because of the hydrophobic nature of trichome (Brewer \& Smith, 1997).

The condensation behavior of the sub-micro scale water droplets generated by molecular-size water vapor on $P$. tomentosum leaves was investigated (Fig. 3). We used ESEM (Fig. 3A) in combination with a Peltier cooling stage (Fig. 3B) to control the temperature and pressure inducing water droplet condensation under supersaturation in an environmentally controlled chamber. By maintaining the temperature of at $2^{\circ} \mathrm{C}$ (corresponding to a water vapor pressure of 5.3 Torr) using the Peltier cooling stage, the chamber pressure was increased from 3 to 5.6 Torr, to initiate water condensation. As shown in Fig. 3C, trichome on the P. tomentosum leaf remained dry, without any water droplets at chamber pressures lower than 5.6 Torr. However, once the chamber pressure reached 5.6 Torr, water droplets $4.5 \sim 5.5 \mu \mathrm{m}$ in diameter appeared at a relatively small contact angle $\left(\mathrm{ca} .40^{\circ}\right)$ and remained attached to the surface of trichome. Thus, the micro-scale condensed water droplets attached to trichome and wet the $P$. tomentosum leaf despite the formation of relatively large scale water drop such as rain or mist hardly wet the $P$. tomentosum leaf). Finally, water flooding occurred, and nearly the entire surface of the leaf as well as trichome was covered 

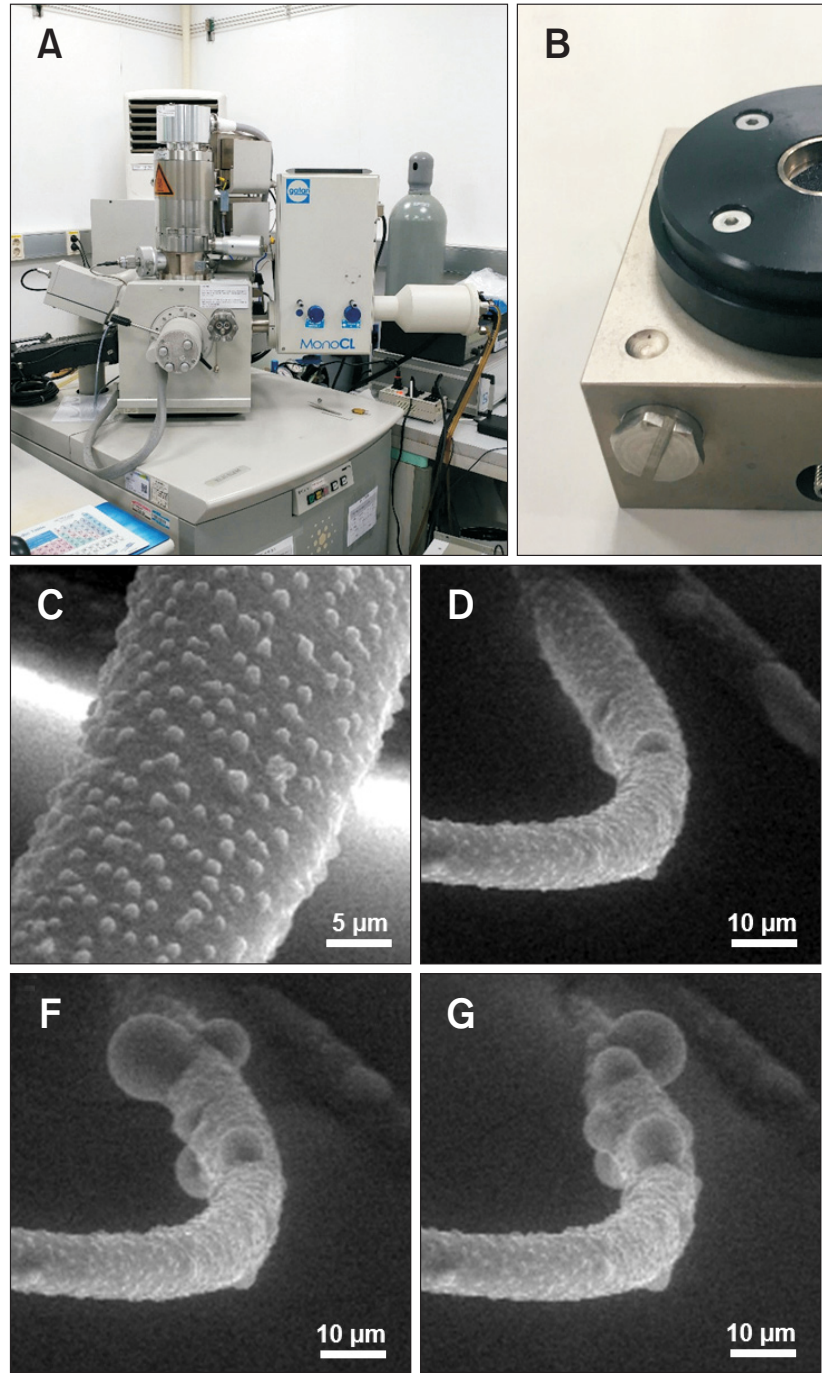

by condensed water with the passage (Fig. 3H).

\section{CONCLUSIONS}

We observed the morphology and water wettability characteristics of $P$. tomentosum leaves and found that the entire surfaces of the leaves were covered with trichome, which comprised a hierarchical structure of the body and bumps. This hydrophobic trichome was responsible for the superhydrophobicity of $P$. tomentosum leaves for millimeter and micro-meter water droplets such as rain drops and mist, respectively. However, ESEM observation of water condensation from molecular-size water vapor revealed that nucleation started on trichome, to which condensed water droplets $4.5 \sim 5.5 \mu \mathrm{m}$ in diameter were attached. Finally, the entire surface, including trichome, was covered by condensed water when the flooding stage was reached.

\section{CONFLICT OF INTEREST}

No potential conflict of interest relevant to this article was reported.

\section{ACKNOWLEDGMENTS}

The authors acknowledge a grant (MPSS-CG-2016-02) from the Disaster and Safety Management Institute funded by the Ministry of Public Safety and Security of Korean government. 


\section{REFERENCES}

Bhushan B and Her E K (2010) Fabrication of superhydrophobic surfaces with high and low adhesion inspired from rose petal. Langmuir 26, 8207-8217.

Brewer C A and Smith W K (1997) Patterns of leaf surface wetness for montane and subalpine plants. Plant Cell Environ. 20, 1-11.

Cha T G, Yi J W, Moon M W, Lee K R, and Kim H Y (2010) Nanoscale patterning of microtextured surfaces to control superhydrophobic robustness. Langmuir 26, 8319-8326.

Cheng Y T, Rodak D E, Angelopoulos A, and Gacek T (2005) Microscopic observations of condensation of water on lotus leaves. Appl. Phys. Lett. 87, 194112.

Cho W K and Choi I S (2008) Fabrication of hairy polymeric films inspired by Geckos: wetting and high adhesion properties. Adv. Funct. Mater. 18, 1089-1096.

Donald A M (2003) The use of environmental scanning electron microscopy for imaging wet and insulating materials. Nat. Mater. 2 511-516.

Esmaily M, Mortazavi N, Shahabi-Navid M, Svensson J E, Johansson L G, and Halvarsson M (2015) On the capability of in-situ exposure in an environmental scanning electron microscope for investigating the atmospheric corrosion of magnesium. Ultramicroscopy 153, 45-54.

Gao X and Jiang L (2004) Biophysics: water-repellent legs of water striders. Nature 432, 36.

Gorb E V and Gorb S N (2002) Attachment ability of the beetle Chrysolina fastuosa on various plant surfaces. Entomol. Exp. Appl. 105, 13-28.

Hansen W R and Autumn K (2005) Evidence for self-cleaning in gecko setae. Proc. Natl. Acad. Sci. U S A 102, 385-389.
Jansson A, Nafari A, Sanz-velasco A, Svensson K, Gustafsson S Hermansson A, and Olsson E (2016) Microscopy microanalysis novel method for controlled wetting of materials in the environmental scanning electron microscope. Microsc. Microanal. 19, 30-37.

Ko T J, Her E K, Shin B, Kim H Y, Lee K R, Hong B K, Kim S H, Oh K H, and Moon M W (2012) Water condensation behavior on the surface of a network of superhydrophobic carbon fibers with high-aspect-ratio nanostructures. Carbon 50, 5085.

Ko T J, Kim S H, Hong B K, Lee K R, Oh K H, and Moon M W (2015) High performance gas diffusion layer with hydrophobic nanolayer under a supersaturated operation condition for fuel cells. ACS Appl. Mater. Interfaces 7, 5506-5513.

Koch K and Barthlott W (2009) Superhydrophobic and superhydrophilic plant surfaces: an inspiration for biomimetic materials. Philos. Trans. Ser. A. Math. Phys. Eng. Sci. 367, 1487-1509.

Neinhuis C and Barthlott W (1997) Characterization and distribution of water-repellent, self-cleaning plant surfaces. Ann. Bot. 79, 667-677.

Quéré D (2008) Wetting and roughness. Annu. Rev. Mater. Res. 38, 71 99.

Rahmawan Y, Moon M W, Kim K S, Lee K R, and Suh K Y (2010) Wrinkled, dual-scale structures of diamond-like carbon (DLC) for superhydrophobicity. Langmuir 26, 484-491.

Shin B, Lee K R, Moon M W, and Kim H Y (2012) Extreme water repellency of nanostructured low-surface-energy non-woven fabrics. Soft Matter. 8, 1817-1823.

Varanasi K K, Hsu M, Bhate N, Yang W, and Deng T (2009) Spatial control in the heterogeneous nucleation of water. Appl. Phys. Lett. 95, 94101 